The Kaplan-Meier method, log-rank test and Cox proportional hazards model were used to estimate the cumulative incidence of clinical disability and perform univariate analysis and multivariate analysis, respectively.

Results $143(44.8 \%)$ out of the 319 CD patients (median follow-up time 37.2 months, interquartile range: 22.7-57.6 months) progressed to clinical disability, and 51 (16.0\%) suffered disability recurrence. Additionally, the cumulative rates of disabling CD were $18.8 \%, 40.1 \%, 55.5 \%, 85.6 \%$ at 1,3 , 5 , 10 years after diagnosis respectively [figure 1]. All disabling events, except adverse steroid event $(p=0.143)$, were significantly fewer $(p=0.018)$ in $\mathrm{MH}$ patients than those without MH. Multivariable analysis revealed the independent protective factor for the occurrence of disability was $\mathrm{MH}$ before disability (HR:0.197; 95\% CI: 0.120-0.325), while penetrating (B3) disease behaviour (HR:2.045; 95\% CI: 1.283-3.259) and ileocolonic (L3) disease location (HR:1.827; 95\% CI: 1.2512.667) at diagnosis were independent risk factors. Furthermore, in disabling $\mathrm{CD}$ patients, achieving $\mathrm{MH}$ before the first disabling event could prevent recurrence of disability independently (HR:0.251; 95\% CI: 0.077-0.823).

IDDF2018-ABS-0246 Figure 1. The cumulative rate of remaining free from disabling CD.

Conclusions Clinical disability of CD was common and increasing during the follow-up in China. B3 and L3 at diagnosis were predictive risk factors of clinical disability, whereas $\mathrm{MH}$ significantly reduced the occurrence and recurrence of disability in CD.

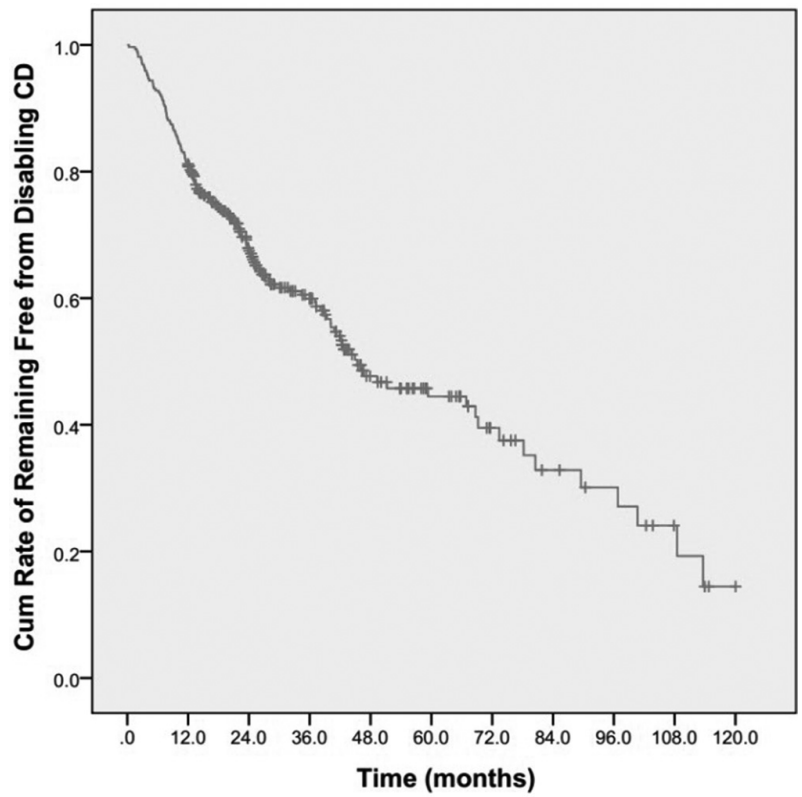

Abstract IDDF2018-ABS-0246 Figure 1

\section{IDDF2018-ABS-0248 EFFICACY OF A NOVEL DISPOSABLE SHEATHED GASTROSCOPIC SYSTEM IN ENDOSCOPIC THERAPY}

Chen $\mathrm{Xi}^{*}$, Jin Peng, Wang Xin, Yu Dongliang, Sheng Jianqiu. Department of Gastroenterology, Beijing Military General Hospital, Beijing, China

10.1136/gutjnl-2018-IDDFabstracts. 172
Background Endoscopic examination and therapy carry a potential risk of cross-infection, and the traditional reprocessing method is time-consuming. Here we reported a novel disposable sheathed gastroscope system and evaluated its efficacy in endoscopic therapy.

Methods The disposable sheathed gastroscope system used in this study consisted of a disposable sheath and a common video gastroscope, which were both provided by Shenda Endoscope Co., Ltd., Shenyang, China. The sterilised disposable sheath covered the outer surface of the gastroendoscope. An outlaid disposable air-water pipe (still covered by the sheath) and a miniature pump instead of the gastroscopic airwater channel were used for sending air and water. A flexible pipe, with one end tightly connected to the distal tip of covering film, was inserted into the gastroscopic suction-biopsy channel and joined to a three-way cap. This pipe was used as the disposable suction-biopsy channel. After examination and therapy, the contaminated sheath, including pipes and valves, was taken off and a new sheath was put on. Using this sheathed gastroscope system, 78 patients with gastric hyperplastic polyps underwent endoscopic mucosal resection in Beijing Military General Hospital. As a control, 76 patients with similar polyps underwent endoscopic resection with a conventional gastroscope. The time duration of the endoscopic procedure and reprocessing were measured.

Results All 154 procedures were completed by using each gastroscope system. No complications were seen during or after the procedures. The endoscopists who performed the procedures felt the sheath did not affect the operation at all, and all endoscopists had no preference for sheathed versus conventional gastroscopes in endoscopic procedures. The mean procedural time was slightly longer in the Sheathed Group than in the Conventional Group $(8.9 \pm 2.2$ vs. $8.1 \pm 1.5$ mins, $\mathrm{p}=0.000)$. However, the duration of endoscopic reprocessing was much shorter $(4.9 \pm 0.2$ vs. $35 \pm 0.2$ mins, $p=0.000)$. No microbial contamination was detected in each gastroscope after procedures.

Conclusions The novel disposable sheathed gastroscope should be suitable for endoscopic therapy.

\section{IDDF2018-ABS-0250 DETERMINANTS OF DEPRESSION AND ITS IMPACT ON QUALITY OF LIFE IN PATIENTS WITH CHRONIC PANCREATITIS}

Subhaleena Sarkar*, Nageshwar Reddy, Rupjyoti Talukdar. Asian Institute of Gastroenterology, India

\subsection{6/gutjnl-2018-IDDFabstracts.173}

Background We evaluated the prevalence of depression and its impact on quality of life(QOL) in CP.

Methods Consecutive patients with CP were screened from Aug-17'-Jan-18'. Following were recorded: demography (age/ gender/education/socioeconomic status); past $3 \mathrm{mths}$ pain details (episodes/intensity[VAS]/severity[mild/moderate/severe]/nature [intermittent/continuous]); other parameters (aetiology/duration/ diabetes/weight loss/morphology[pancreatic duct size/calculi/calcifications]; and treatment details (diet/medical/interventional/ compliance).

Depression was measured using Beck's Depression Inventory (BDI); QOL using QORTC-QLQ30 + PAN28 tool. 